\title{
THE ASSOCIATION BETWEEN TRAIT FORGIVENESS AND MENTAL HEALTH: THE MEDIATING EFFECT OF RUMINATION
}

\author{
Wei $\mathrm{Li}^{1 *}$, Xiaofeng Yang ${ }^{2}$ \\ ${ }^{1}$ Associate Prof. Dr. Mrs., Inner Mongolia Normal University, PR China, lily2669@hotmail.com \\ ${ }^{2}$ Associate Prof. Dr. Mr., Inner Mongolia Normal University, PR China, steveyangxf@hotmail.com \\ ${ }^{*}$ Corresponding author
}

\begin{abstract}
Trait forgiveness is a tendency to forgive others across a wide range of interpersonal circumstances. Though there is growing evidence to suggest that failure to forgive is closely related to indicators of poor mental health as depression, anxiety and anger, less attention has been paid to the relationship between trait forgiveness and mental health. In order to study the mechanism of the relationship of these two variables, we introduce rumination as a third variable, which has been proved to correlate negatively with state forgiveness and positively with mental health problems. We hypothesized that an individual's mental health could be predicted by his/her trait forgiveness and rumination. We tested this hypothesis using a survey, in which five hundred and thirty-seven college students (175 males and 362 females) from five universities in China were investigated with Trait Forgiveness Scale (TFS), Ruminative Response Scale (RRS), Centre for Epidemiological Studies-Depression Scale (CES-D), Self-Rating Anxiety Scale (SAS) and Trait Anger Scale (TAS). Descriptive statistics and inferential statistics were adopted to analyse the data of survey with SPSS 21. Results indicate that (a) significant gender difference exists in trait forgiveness, that is, trait forgiveness of female students is significantly higher than that of male students $(t=-2.06, p<0.05)$; (b) students in Low Forgiveness Group (the Lower 27\%) have higher scores of depression, anxiety and anger than those in High Forgiveness Group (the Upper 27\%) $(t=-5.86,-6.07$ and -7.48 , respectively, $p s<0.001$ ); (c) trait forgiveness negatively correlates with rumination $(r=-0.21, p<0.001)$, depression $(r=-0.30, p<0.001)$, anxiety $(r=-0.31, p<0.001)$ and anger $(r=-0.34, p<0.001)$, while rumination positively correlates with depression $(r=0.61, p<0.001)$, anxiety $(r=0.55, p<0.001)$ and anger $(r=0.36, p<0.001)$; (d) among the predictive variables, trait forgiveness is the best one, which accounts for $8.7 \%, 9.5 \%$ and $11.6 \%$ of the variation in depression, anxiety and anger; (e) rumination serves as a partial mediator variable between trait forgiveness and mental health. These results imply that although trait forgiveness and rumination both affect mental health, the affecting paths were different. Trait forgiveness may affect mental health directly, or indirectly through rumination.
\end{abstract}

Keywords: trait forgiveness, mental health, rumination, mediating effect

\section{INTRODUCTION}

The construct of forgiveness has received increasing attention in the past few years and was defined from different perspectives (McCullough \& Witvliet, 2002). As a response, forgiveness can be defined as a freely made choice to give up revenge, resentment, or harsh judgments toward a person who caused a hurt, and to strive to respond with generosity, compassion, and kindness toward that person (Enright, Freedman, \& 
Rique, 1998). It is a process that involves reducing negative responses and increasing positive responses toward the person who caused the hurt, across the realms of affect, cognition, and behavior (Enright \& Fitzgibbons, 2015). As a personality disposition, forgiveness can be conceptualized as a tendency to forgive others across a wide range of interpersonal circumstances. In this sense, people can be scaled along a forgiving-unforgiving continuum, with most people falling somewhere toward mean of the population (Enright et al., 1998). Individuals who are more forgiving may be accustomed to choosing forgiving others as their habitual response to interpersonal transgressions across the time and situations (Berry, Worthington, Parrott, O Connor, \& Wade, 2001; Yang, 2013). The response-based forgiveness can be classified as state forgiveness while the disposition-based forgiveness can be classified as trait forgiveness.

A variety of empirical studies have confirmed the close association between state forgiveness and mental health. State forgiveness is negatively associated with depression, anxiety (Freedman \& Enright, 1996;

Reed \& Enright, 2006), anger (Lawler-Row, Karremans, Scott, Edlis-Matityahou, \& Edwards, 2008) and even suicidal behaviors (Hirsch, Webb, \& Jeglic, 2011). Researchers differ on the mechanisms underlying the relationship between forgiveness and mental health. So far, It is generally believed that forgiveness can affect mental health both directly and indirectly (Webb, Robinson, \& Brower, 2011). The former mainly explores the roles of forgiveness on suppressing negative emotions and promoting subject well-being, while the latter mainly focuses on the mediating or moderating variables between forgiveness and mental health, such as stress, social support and health behaviors (Zhang \& Wu, 2012).

Early studies have found that rumination is an important influencing factor of forgiveness. Rumination is a mode of responding to distress that involves repetitively and passively focusing on symptoms of distress and on the possible causes and consequences of these symptoms (Nolen-Hoeksema, Wisco, \& Lyubomirsky, 2008). People who are ruminating just remain fixated on the problems and on their feelings about them without taking actions to solve them. According to Nolen-Hoeksema et al. (2008), rumination is associated with psychopathologies in addition to depression, including anxiety, binge eating, binge drinking, and selfharm. In the context of interpersonal transgressions, victims are likely to ruminate about the offense and the corresponding negative emotions. An inverse relationship between forgiveness and rumination has been found in different samples from the United States (McCullough, Bono, \& Root, 2007), India (Suchday, Friedberg, \& Almeida, 2006) and Italy (Paleari, Regalia, \& Fincham, 2005).

Though there is growing evidence to suggest that failure to forgive is closely related to indicators of poor mental health as depression, anxiety and anger, less attention has been paid to the relationship between trait forgiveness and mental health (Sandage \& Jankowski, 2010). The relationship between trait forgiveness and rumination also needs further exploring. The present study assumes that rumination may serve as a mediator between trait forgiveness and mental health. The exploring of the relationship between the variables among Chinese undergraduates will, thus, make a unique contribution to the literature.

\section{METHOD}

\subsection{Participants and Procedure}

A sample of 537 college students (175 men and 362 women) with a mean age of 19.82 years (SD =1.67) participated in the study. Students from five universities of three cities in China, namely Hohhot, Beijing and Guangzhou, were drawn as a convenience sample. The students were told by the instructors to participate in the study voluntarily without receiving any extra credit and to complete the scales anonymously in class. Measures of forgiveness, mental health and rumination were completed as part of a standard intake battery of assessments. All participants were treated in accordance with the ethical guidelines of the American Psychological Association.

\subsection{Measures}

\subsubsection{The Trait Forgiveness Scale (TFS )}

This 8-item scale measures two dimensions of trait forgiveness: tendency to forgive (TF) and tendency to revenge (TR). Each subscale includes four items. The scale was developed on the basis of previous literature review, open questionnaire survey and individual interviews (Yang, 2013). Respondents are required to rate the items on a 7-point Likert-type scale ranging from 1 (strongly disagree) to 7 (strongly agree). The TFS has shown acceptable internal consistency reliability (TF: $\alpha=0.74$; TR: $\alpha=0.75$ ) and testretest reliability (TF: $r s=0.71$; TR: $r s=0.74$ for 4 weeks intervals). The TFS has also shown acceptable construct validity supported by confirmatory factor analysis (CFA) and convergent and divergent correlations with other measures of forgiveness and related constructs. 


\subsubsection{The Ruminative Response Scale (RRS)}

The RRS is a 22-item, 4-point Likert-type scale ( 4 = almost always; 1 = almost never) self-report instrument which is designed to measure the tendency to ruminate in response to feelings of sadness and depression. It contains three subscales named Symptom Rumination, Brooding and Reflective Pondering. The total score of the RRS is the sum of scores on the 22 items. The higher the total score, the more serious the tendency to ruminate. Both original version and Chinese version showed acceptable psychometric properties (Han \& Yang, 2009). The structural validity of the RRS was verified with the fitting degree of theoretical model and actual data with the software of Amos 21.0. The model structure was further adjusted according to the modified index and 4 items were deleted. Results showed that the modified model had better fitting indexes: $d f=128, X^{2} / d f=2.14, R M S E A=0.046, G F I=0.95, A G F I=0.93, C F I=095, N F I=0.91$. The revised 18-item RRS is still a three-factor structure. The internal consistency coefficients of the total scale and subscale were $0.89,0.86,0.73$ and 0.71 , respectively.

\subsubsection{The Centre for Epidemiological Studies-Depression (CES-D)}

The CES-D scale, originally published by Radloff (1977), is a 20-item self-report scale designed to measure depressive symptomatology in the general population. Respondents are asked to indicate how often over the past week they experienced symptoms associated with depression. Response options range from 1 to 4 for each item $(1=$ Rarely or None of the Time, $2=$ Some or Little of the Time, $3=$ Moderately or Much of the time, $4=$ Most or Almost All the Time). Total scores range from 20 to 80 , with higher scores indicating greater depressive symptoms. The internal consistency coefficient of the scale in this study was 0.90 .

\subsubsection{The Self-rating anxiety scale (SAS)}

The SAS, originally invented by Zung (1971), is a 20 -item well-accepted self-report assessment device built to measure anxiety levels in both clinical and research settings. Respondents are asked to indicate how much each statement applies to him/her during the past one or two weeks. Each item is scored on a 4-points Likert-type scale ( 1 = Rarely or None of the Time, $2=$ Some or Little of the Time, $3=$ Moderately or Much of the time, $4=$ Most or Almost All the Time). Five out of twenty items are negatively worded. The total scores range from 20 to 80 . The internal consistency coefficient of the Chinese version of SAS in present study was 0.90 .

\subsubsection{The Trait Anger Scale (TAS)}

The TAS, a subscale of the state-trait anger expression scale (STAXI) invented by Spielberger (1988), is a 10-item Likert-type scale ( $1=$ almost never, $4=a$ almost always) which is used to assess general temperament and responses to certain situations. Total scores range from 10 to 40 with higher scores indicating a greater tendency to perceive more situations as anger-provoking and to experience heightened state anger. According to Luo, Zhang, Liu, and Liu (2011), the Chinese version of the TAS had good reliability and validity and could be used to assess trait anger in college students.

\section{RESULTS}

\subsection{Independent Sample T-test}

The study firstly examined whether there was any difference in demographic variables. Independent sample T-test showed that trait forgiveness of female students was significantly higher than that of male students $(t=$ $-2.06, p<0.05)$.

Then, according to the score of trait forgiveness, the respondents were divided into two groups: high score group (the first 27\%) and low score group (the last 27\%). Independent sample T-test was used to compare the differences in mental health between two groups. Results showed that significant differences existed in depression $(t=-5.86, p<0.001)$, anxiety $(t=-6.07, p<0.001)$ and anger $(t=-7.48, p<0.001)$. The individual who has higher score of trait forgiveness scores less in depression, anxiety and anger, which indicates a better mental health status.

\subsection{Partial Correlation Analysis}

Considering the demographic variables may interact with trait forgiveness, in turn, impact on mental health, the study adopted partial correlation analysis to examine the association between trait forgiveness and mental health under the premise of controlling demographic variables. Results showed trait forgiveness was still negatively correlated with depression $(r=-0.30, p<0.001)$, anxiety $(t=-0.31, p<0.001)$ and anger $(t=-$ $0.34, p<0.001)$. 


\subsection{Multivariate Regression Analysis}

Multivariate regression analyses with Stepwise method were conducted to further explore the association among demographic variables, trait forgiveness and mental health, among which gender, major, origin, siblings number and trait forgiveness were set as predicative variables, while depression, anxiety and anger as dependent variables. Results showed that (1) trait forgiveness and siblings number could predict depression significantly and together they could account for $9.6 \%$ of the variation; (2) trait forgiveness and siblings number could predict anxiety significantly and together they could account for $10.4 \%$ of the variation; (3) trait forgiveness itself could predict anger significantly and it could account for $11.6 \%$ of the variation; (4) in the above three regression analyses, trait forgiveness was the best predictive variable, and it could account for $8.7 \%, 9.5 \%$ and $11.6 \%$ of the variation in depression, anxiety and anger (See Table 1).

Table 1 Results of Multivariate Regression Analysis

\begin{tabular}{|c|c|c|c|c|c|c|c|}
\hline DV & IV & $\boldsymbol{R}$ & $R 2$ & $\Delta \boldsymbol{R}$ & $\boldsymbol{F}$ & Beta & $t$ \\
\hline \multirow{2}{*}{ Depression } & Trait forgiveness & 0.295 & 0.087 & 0.087 & $51.063^{\star \star}$ & -0.302 & $-7.325^{*}$ \\
\hline & Siblings number & 0.310 & 0.096 & 0.009 & $28.310^{\star \star \star}$ & 0.094 & 2.272 \\
\hline \multirow{2}{*}{ Anxiety } & Trait forgiveness & 0.307 & 0.095 & 0.095 & $55.857^{\star \star \star}$ & -0.315 & $-7.669^{*}$ \\
\hline & Siblings number & 0.323 & 0.104 & 0.009 & $31.107^{\star \star \star}$ & 0.099 & 2.419 \\
\hline Anger & Trait forgiveness & 0.340 & 0.116 & 0.116 & $70.080^{* *}$ & -0.340 & $-8.371^{*}$ \\
\hline
\end{tabular}

Note. ${ }^{*} p<0.05,{ }^{* *} p<0.01,{ }^{* \star *} p<0.001$; IV=independent variable; $\mathrm{DV}=$ dependent variable

\subsection{Pearson Correlation Analysis}

The correlations between rumination, trait forgiveness and mental health (including depression, anxiety and anger) were calculated (see Table 2). Result showed that all variables were significantly correlated (all $p<$ 0.001): (1) Rumination correlated negatively with trait forgiveness, but positively with mental health (including depression, anxiety and anger); (2) Trait forgiveness correlated negatively with rumination and mental health (including depression, anxiety and anger); (3) Mental health (including depression, anxiety and anger) correlated positively with rumination but negatively with trait forgiveness. That is to say, individuals who are more forgiving generally have less rumination and less mental health problems.

Table 2 Correlations between trait rumination, forgiveness and mental health

\begin{tabular}{|c|c|c|c|c|c|}
\hline & Rumination & Anxiety & Depression & Anger & Trait Forgiveness \\
\hline Rumination & 1.00 & & & & \\
\hline Anxiety & $0.55^{\circ}$ & 1.00 & & & \\
\hline Depression & 0.61 & 0.77 & 1.00 & & \\
\hline Anger & 0.36 & 0.40 & $\overline{0.42}$ & 1.00 & \\
\hline Trait Forgiveness & $-0.21^{*}$ & $-0.31^{*}$ & $-0.30^{\circ}$ & $-0.34^{\prime \prime}$ & 1.00 \\
\hline
\end{tabular}

Note. ${ }^{\star} p<0.05,{ }^{* \star} p<0.01,{ }^{* * *} p<0.001$

\subsection{Mediating Effects Testing}

Based on the comparison of various testing methods of mediating effects, Wen and Ye (2014) proposed a composite testing procedure, which was better than any single testing method in terms of type 1 and type 2 error rates and could be used to test both partial and full mediating effects. According to Wen's newly proposed procedure, three regression equation models were run to estimate the relationships among rumination, trait forgiveness and depression and to generate the statistics needed to test the mediating effects (see Table 3). In equation \# 1, trait forgiveness was regressed on depression to estimate the regression coefficient $c\left(\beta_{c}=-0.295, p<0.001\right)$. In equation $\# 2$, trait forgiveness was regressed on rumination to estimate the regression coefficient a $\left(\beta_{a}=-0.214, p<0.001\right)$.In equation \# 3 , both trait forgiveness and rumination were regressed on depression to estimate the regression coefficient c' and b ( $\beta_{c^{\prime}}=-0.172, \beta_{b}=-0.575, p s<0.001$ ). The regression coefficients a and $b$ were significant, indicating that rumination was the mediator between trait forgiveness and depression, while the regression coefficients $c$ and c' were significant, indicating that rumination had a partial mediating effect between trait forgiveness and depression (Wen, Chang, Hau, \& Liu, 2004; Wen \& Ye, 2014). In present study, the coefficient measuring 
the total effect of trait forgiveness on depression was -0.295 in equation \# 1 . When rumination was introduced in equation \# 3 , the coefficient was reduced to -0.172 , indicating that rumination mediated the relationship between trait forgiveness and depression. The decrease of $0.028(0.403-0.375)$ in the standardized coefficient represented the mediated effect, and the ratio of mediating effects on total effects was $41.7 \%\left(\right.$ Effect $\left._{\mathrm{m}}=\mathrm{ab} / \mathrm{c}=0.214 \times 0.575 \div 0.295=0.417\right)$.

The similar mediating effect of rumination was also found between trait forgiveness and anxiety, trait forgiveness and anger. Due to the limitations in space, the statistics are not presented here. The mediating effects of rumination between trait forgiveness and mental health (including depression, anxiety and anger) could be depicted through a path diagram (see Fig. 1).

Table 3 Mediating effect of Rumination between Trait Forgiveness and Depression

\begin{tabular}{|l|l|l|l|l|l|l|l|}
\hline Equation & $\mathrm{IV} \rightarrow \mathrm{DV}$ & $R$ & $R^{2}$ & $\Delta R$ & $F$ & Beta & $t$ \\
\hline$(1) \mathrm{Y}=\mathrm{cX}+\mathrm{e}_{1}$ & $\mathrm{TF} \rightarrow \mathrm{D}$ & 0.295 & 0.087 & 0.087 & 51.063 & -0.295 & -7.146 \\
\hline$(2) \mathrm{M}=\mathrm{aX}+\mathrm{e}_{2}$ & $\mathrm{TF} \rightarrow \mathrm{R}$ & 0.214 & 0.046 & 0.046 & 25.552 & -0.214 & -5.055 \\
\hline \multirow{2}{*}{$(3) \mathrm{Y}=\mathrm{c}^{\prime} \mathrm{X}+\mathrm{bM}+\mathrm{e}_{3}$} & $\mathrm{R} \rightarrow \mathrm{D}$ & 0.612 & 0.375 & 0.375 & $320.381^{\prime \prime}$ & 0.575 & $16.805^{\prime \prime \prime}$ \\
\cline { 2 - 8 } & $\mathrm{TF} \rightarrow \mathrm{D}$ & 0.635 & 0.403 & 0.028 & $180.164^{\prime \prime \prime}$ & -0.172 & -5.036 \\
\hline
\end{tabular}

Note. ${ }^{* * *} p<0.001 ; \mathrm{IV}=$ independent variable; $\mathrm{DV}=$ dependent variable; TF $=$ Trait forgiveness; $\mathrm{R}=$ rumination; $\mathrm{D}$ = depression;

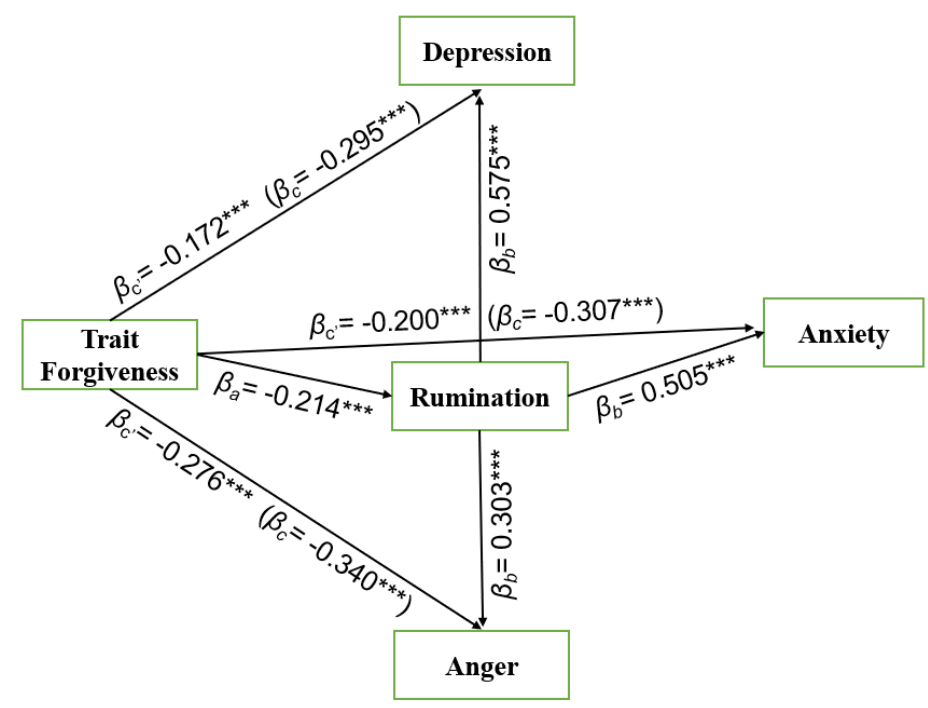

Fig.1 Path program of mediating effects

\section{DISCUSSION}

In this study, the gender difference of trait forgiveness was found, namely, the score of trait forgiveness in female students was significantly higher than that in male students, which was consistent with most researches (Brown, 2003; Li, 2011). The gender differences may be related to the different levels of empathy, the different thinking styles or the different tendencies in moral choice and moral judgment between male and female.

As for the relationship between trait forgiveness and mental health, we hypothesized that two paths existed: direct path and indirect path. On one hand, trait forgiveness may directly promote mental health. On the other hand, trait forgiveness may indirectly influence mental health through some mediating variables. The results of this study can be used to verify the existence of these two paths.

Results of Independent sample $T$-test, partial correlation analysis and regression analysis showed that students in low forgiveness group had significantly higher scores in depression, anxiety and anger than those in high forgiveness group. Trait forgiveness negatively correlated with depression, anxiety and anger and could predict mental health significantly. This result is consistent with the universally accepted conclusion that forgiveness is negatively correlated with the bad health indicators like dysfunction or pain (Tse \& Yip, 2009). As a self-protection mechanism, trait forgiveness can help individuals release negative 
emotions such as anger and hatred and maintain their physical and mental health. Less forgiving but more revengeful individuals, who are always under tremendous physical and mental pressures, frequently experience bad emotions like anger, hostility, dismay, anxiety and inferiority, which may result in depressed moods, dysfunctional immune system (Seybold, Hill, Neumann, \& Chi, 2001), high blood pressure or heart diseases (Friedberg, Suchday, \& Shelov, 2007). Apparently, trait forgiveness can affect individual mental health directly.

The partial mediating effect of rumination between trait forgiveness and mental health was also found in present study. The strong link between rumination and forgiveness has been proved well in previous studies. On one hand, rumination is one of the important factors that influence forgiveness. When a person is treated unfairly or at times cruelly, he might constantly recall, brood and focus on the injustice and repeatedly experience negative emotions, which will unconsciously result in avoidance and revenge motivations. When the offended tries to suppress or avoid these motivations, he may ruminate the offense and further enhance these motivations. This vicious circle will seriously affect individual's choice to forgive (McCullough, Bellah, Kilpatrick, \& Johnson, 2001). On the other hand, trait forgiveness, in turn, will affect individual's tendency of rumination. People with more trait forgiveness get accustomed to choosing forgiving others as their habitual response to interpersonal transgressions across the time and situations (Yang, 2013). They don't think it is necessary to ruminate upon the offenses repeatedly and will not be troubled too much by them. In contrast, people with less trait forgiveness tend to adopt emotion-centered strategies. They ruminate a lot on the offenses and enhance the avoidance or revenge motivation to the wrongdoers, which may leave them in poor health (McCullough et al., 2001; Seybold, Hill, Neumann, \& Chi, 2001). An investigation conducted by Friedberg, Adonis, Von Bergen, and Suchday (2005) on New Yorkers one year after the September 11th terrorist attack also proved the existence of indirect mechanism that rumination mediated the relationship between forgiveness and perceived stress. Forgiveness appears to serve as a buffer against mental health problems more in individuals with low levels of rumination than in individuals with high levels of rumination.

\section{CONCLUSION}

(1) Significant gender differences existed in trait forgiveness, that is, trait forgiveness of female students was significantly higher than that of male students.

(2) Trait forgiveness negatively correlated with depression, anxiety and anger.

(3) Trait forgiveness could predict mental health significantly.

(4) Rumination served as a partial mediating variable between trait forgiveness and mental health.

\section{REFERENCE LIST}

Berry, J. W., Worthington, E. L., Parrott, L., O Connor, L. E., \& Wade, N. G. (2001). Dispositional forgivingness: Development and construct validity of the Transgression Narrative Test of Forgivingness (TNTF). Personality and Social Psychology Bulletin, 27(10), 1277-1290.

Brown, R. P. (2003). Measuring individual differences in the tendency to forgive: Construct validity and links with depression. Personality and Social Psychology Bulletin, 29(6), 759-771.

Enright, R. D., \& Fitzgibbons, R. P. (2015). Forgiveness therapy: An empirical guide for resolving anger and restoring hope: American Psychological Association.

Enright, R. D., Freedman, S., \& Rique, J. (1998). The psychology of interpersonal forgiveness. In D. Tutu, R. D. Enright, \& J. North (Eds.), Exploring forgiveness (pp. 46-62). Madison: University of Wisconsin Press.

Freedman, S. R., \& Enright, R. D. (1996). Forgiveness as an intervention goal with incest survivors. Journal of Consulting and Clinical Psychology, 64(5), 983-992.

Friedberg, J. P., Adonis, M. N., Von Bergen, H. A., \& Suchday, S. (2005). Short communication: September 11 th related stress and trauma in New Yorkers. Stress and Health, 21(1), 53-60.

Han, X., \& Yang, H. (2009). Chinese version of Nolen-Hoeksema Ruminative Responses Scale (RRS) used in 912 college students : Reliability and validity. Chinese Journal of Clinical Psychology, 17(05), 550551. 
Hirsch, J. K., Webb, J. R., \& Jeglic, E. L. (2011). Forgiveness, depression, and suicidal behavior among a diverse sample of college students. Journal of Clinical Psychology, 67(9), 896-906.

Lawler-Row, K. A., Karremans, J. C., Scott, C., Edlis-Matityahou, M., \& Edwards, L. (2008). Forgiveness, physiological reactivity and health: the role of anger. International Journal of Psychophysiology, 68(1), 51-58.

$\mathrm{Li}, \mathrm{X}$. (2011). Study on the forgiveness and its correlation with mental health among college students. Modern Preventive Medicine, 38(14), 2763-2766.

Luo, Y., Zhang, D., Liu, Y., \& Liu, Y. (2011). Reliability and validity of the Chinese version of Trait Anger Scale in college students. Chinese Mental Health Journal, 25(9), 700-704.

McCullough, M. E., Bellah, C. G., Kilpatrick, S. D., \& Johnson, J. L. (2001). Vengefulness: Relationships with forgiveness, rumination, well-being, and the Big Five. Personality and Social Psychology Bulletin, 27(5), 601-610.

McCullough, M. E., Bono, G., \& Root, L. M. (2007). Rumination, emotion, and forgiveness: Three longitudinal studies. Journal of Personality and Social Psychology, 92(3), 490-505.

McCullough, M. E., \& Witvliet, C. V. O. (2002). The psychology of forgiveness. In C. R. E. Snyder \& S. J. Lopez (Eds.), Handbook of positive psychology (pp. 446-455). New York: Oxford University Press.

Nolen-Hoeksema, S., Wisco, B. E., \& Lyubomirsky, S. (2008). Rethinking rumination. Perspectives on psychological science, 3(5), 400-424.

Paleari, F. G., Regalia, C., \& Fincham, F. (2005). Marital quality, forgiveness, empathy, and rumination: A longitudinal analysis. Personality and Social Psychology Bulletin, 31(3), 368-378.

Radloff, L. S. (1977). The CES-D scale: A self-report depression scale for research in the general population. Applied psychological measurement, 1(3), 385-401.

Reed, G. L., \& Enright, R. D. (2006). The effects of forgiveness therapy on depression, anxiety, and posttraumatic stress for women after spousal emotional abuse. Journal of Consulting and Clinical Psychology, 74(5), 920-920-929.

Sandage, S. J., \& Jankowski, P. J. (2010). Forgiveness, spiritual instability, mental health symptoms, and well-being: Mediator effects of differentiation of self. Psychology of Religion and Spirituality, 2(3), 168168-180.

Seybold, K. S., Hill, P. C., Neumann, J. K., \& Chi, D. S. (2001). Physiological and psychological correlates of forgiveness. Journal of Psychology and Christianity, 20(3), 250-259.

Spielberger, C. D. (1988). Manual for the state-trait anger inventory (STAXI). Odessa: Psychological assessment resources.

Suchday, S., Friedberg, J. P., \& Almeida, M. (2006). Forgiveness and rumination: a cross-cultural perspective comparing India and the US. Stress and Health, 22(2), 81-89.

Tse, W. S., \& Yip, T. (2009). Relationship among dispositional forgiveness of others, interpersonal adjustment and psychological well-being: Implication for interpersonal theory of depression. Personality and Individual Differences, 46(3), 365-368.

Webb, J. R., Robinson, E. A. R., \& Brower, K. J. (2011). Mental health, not social support, mediates the forgiveness-alcohol outcome relationship. Psychology of Addictive Behaviors, 25(3), 462-473.

Wen, Z., Chang, L., Hau, K.-T., \& Liu, H. (2004). Testing and application of the mediating effects. Acta Psychologica Sinica, 36(5), 614-620.

Wen, Z., \& Ye, B. (2014). Analyses of Mediating Effects: The Development of Methods and Models. Advances in Psychological Science, 22(5), 731-745.

Yang, X., Li, W., \& Zheng, X. (2019). Development of trait forgiveness questionnaire for college students. Psychology: Techniques and Application, 7(8): 472-484.

Zhang, D., \& Wu, Y. (2012). Social support mediating between trait forgiveness and mental health. Chinese Journal of Clinical Psychology, 20(4), 577-579.

Zung, W. W. (1971). A rating instrument for anxiety disorders. Psychosomatics: Journal of Consultation and Liaison Psychiatry, 12(6), 371-379. 